\title{
Neural competition via lateral inhibition between decision processes and not a STOP signal accounts for the antisaccade performance in healthy and schizophrenia subjects
}

\author{
Vassilis Cutsuridis* \\ Institute of Molecular Biology and Biotechnology, Foundation for Research and Technology - Hellas, Heraklion, Greece \\ *Correspondence: vcutsuridis@gmail.com
}

Edited by:

Tobias H. Donner, University of Amsterdam, Netherlands

Reviewed by:

Florian Ostendorf, Charité - Universitätsmedizin Berlin, Germany

Keywords: decision making, neural network model, eye movement, accumulator, superior colliculus, cortex

\section{A commentary on}

Re-starting a neural race: anti-saccade correction

by Noorani, I., and Carpenter, R. H. S. (2014). Eur. J. Neurosci. 39, 159-164. doi: 10.1111/ejn.12396

Decision making is the process of accumulating evidence about the world and the utility of possible outcomes (Cutsuridis, 2010). A paradigm often used by behavioral neuroscientists to investigate decision processes is the antisaccade paradigm (see Figure 1A; Hallett, 1978). In the antisaccade paradigm subjects are required to suppress an erroneous saccade (error prosaccade) toward a peripheral stimulus and instead make an eye movement to a position in the opposite hemifield (antisaccade). The response repertoire of a subject performing the antisaccade task has been reported to be: (1) the subject makes an erroneous response (i.e., looking toward the peripheral stimulus), (2) the subject makes the antisaccade (i.e., looking in the opposite direction of the peripheral stimulus, and (3) the subject makes an erroneous response followed by a corrected antisaccade (Evdokimidis et al., 2002).

Many computer models of decision making have been advanced. In these models, decision making involves a gradual accumulation of evidence with a variable rate $r$. When this accumulation process crosses a threshold $S_{T}$, then a response is generated. Response time (RT) is then the time from the onset of the decision process till when the decision processes crosses $S_{T}$.

Recently the LATER (Linear Approach to Threshold at Ergodic Rate) model has been extended in the realm of the antisaccade task (see Figure 1B): (Noorani and Carpenter, 2014). The model consisted of three accumulator units racing to threshold: an "anti" unit, a "pro" unit, and a "stop" unit. The "stop" unit prevented the "pro" unit from reaching threshold, thus allowing the "anti" unit to reach a different threshold a little later. The authors hypothesized that the threshold level of the "pro" unit was higher than the "anti" unit's threshold, reflecting this way the advice given by the experimenters to every subject to avoid errors. How often the "stop" unit canceled the "pro" unit depended on its rate of accumulation $(\mu)$ and its variance $\left(\sigma^{2}\right)$. In the case the "pro" unit reached the threshold first, it restarted the "anti" unit allowing it to reach the threshold and generate the antisaccade response. The model's performance was contrasted against the performance of five healthy subjects performing the antisaccade task. The model captured most of the response repertoire observed in the antisaccade task, namely the antisaccades and error prosaccades followed by corrected antisaccades, but not the error prosaccades, their corresponding latency distributions and the error response rate. Despite the model's successes, the model had several shortcomings.
First, the model is unable to produce just the error prosaccade. This shortcoming is inherent in the model. The authors postulated that if the STOP signal did not prevent the error prosaccade response, then the "pro" unit will always restart the "anti" unit (Noorani and Carpenter, 2014). This means the error prosaccades followed by corrected antisaccades will always be produced. If the "stop" unit did prevent the "pro" unit, then the "anti" unit would not re-start, and an antisaccade response would be generated (Noorani and Carpenter, 2014). In either scenario, just an error prosaccade response cannot be generated. Psychophysical studies of the antisaccade task (Evdokimidis et al., 2002) have reported that subjects make just erroneous prosaccades, but their response frequency is low.

Furthermore, the model implies that the latency of the corrected antisaccade is the result of the linear sum of latencies of the error prosaccade and the antisaccade minus the latency of the STOP activity. This shortcoming is also inherent in the model, because its units are considered linear encoders of the input information. In contrast, all neurons in the brain (units in the model) non-linearly transform the sum of the dendritic outputs before they generate a neuronal response. Dendritic subunits are perhaps the only linear encoders of incoming information (Polsky et al., 2004).

Moreover, the model postulates the existence of a STOP signal, which occasionally stops the error prosaccade 
A

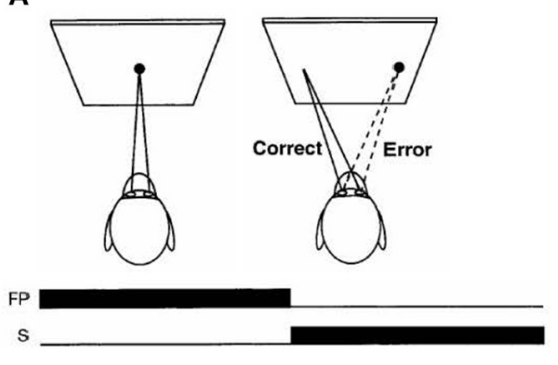

B

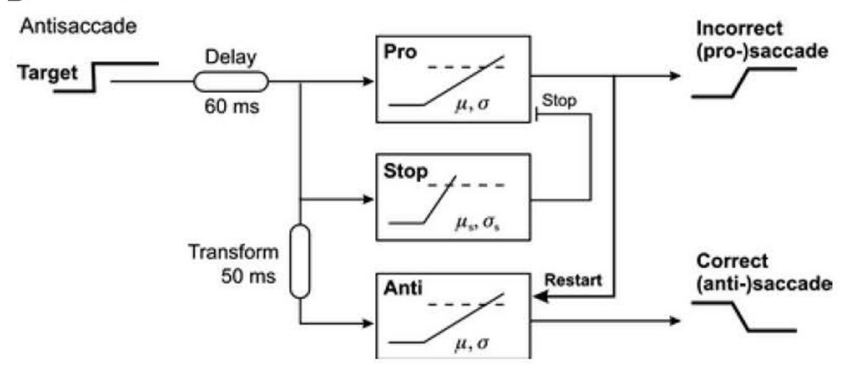

C

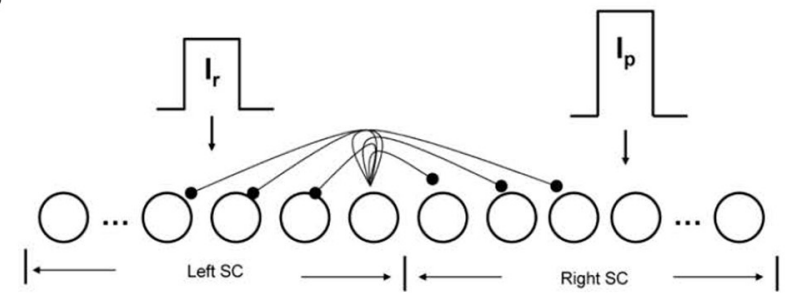

FIGURE 1 | (A) Mirror antisaccade task. (B) Noorani and Carpenter (2014) model for antisaccades (reprinted with permission from Noorani and Carpenter, 2014). (C) Cutsuridis et al. (2014) neural network model of the superior colliculus (SC) for antisaccades in healthy and schizophrenia subjects (reprinted with permission from Cutsuridis et al., 2014). Neurons are represented as nodes. Short-range lateral excitation and long distance lateral inhibition was assumed between all nodes in the network. The left half of the network represented the left SC, whereas the right half represented the right SC. The left SC was activated by a reactive input $I r$ (error prosaccade decision signal), whereas the right SC was activated by a planned input Ip (antisaccade decision signal). The strengths of the inputs were not equal. response and indirectly allows just the antisaccade response to be expressed (Noorani and Carpenter, 2014). Many past experimental studies have speculated the origins of such a signal (basal ganglia, FEF, DLPFC, etc.) (Munoz and Everling, 2004), but recent experimental evidence has challenged the existence of such a signal (Everling and Johnston, 2013). On the other hand, computational studies have suggested that such a STOP signal might operate as a top-down excitatory signal which activates the local inhibitory neurons in a distant area, which in turn inhibit their neighboring excitatory neurons (Brown et al., 2004).

Attractive alternatives of the Noorani and Carpenter (2013, 2014) models of the antisaccade performance are the models of Cutsuridis et al. (2007, 2014) (see Figure 1C). Cutsuridis et al. (2007) proposed that competition via lateral inhibition (Takahashi et al., 2005; Phongphanphanee et al., 2014) between neurons encoding the volitional antisaccade and neurons encoding the erroneous prosaccade is sufficient to accurately reproduce the error rate and antisaccade, error prosaccade and corrected antisaccade latency distributions of antisaccade data from a large cohort of healthy subjects (Evdokimidis et al., 2002). The model's neurons were non-linear accumulators of incoming information and represented the build-up neurons experimentally recorded in the superior colliculus (SC) (Munoz and Wurtz, 1995).

Recently, Cutsuridis et al. (2014) extended their model in the realm of schizophrenia. Their model showed in a quantitative way why the antisaccade performance of schizophrenia patients is so poor. It predicted that this performance is not due to a deficit in the top-down inhibitory control of the erroneous response as many speculated, but instead it is a product of the competition between the neuronal representations of the erroneous prosaccade and antisaccade responses in the superior colliculus. The model was successful at capturing the response repertoire (error rates, the median antisaccade, median error prosaccade and median corrected antisaccade latencies as well as the antisaccade, error prosaccade and corrected antisaccade distributions) of both healthy and schizophrenia subjects [see Figures 1, 3 and 4 in Cutsuridis et al. (2014) study].
Overall, competition via lateral inhibition between non-linear accumulator neurons seems to be a better mechanism than the "stop-and-restart" mechanism of Noorani and Carpenter (2014), because it captures the full antisaccade performance (latencies of error prosaccades, antisaccades and corrected antisaccades and error rates) of healthy and diseased subjects performing the antisaccade task. Other parameters that may affect the antisaccade performance are the differential strengths of the erroneous prosaccade and the volitional antisaccade signals, or different baseline and/or threshold levels. Although recent experimental evidence has just demonstrated that lateral interactions within SC intermediate segment are more suitable for faithfully accumulating subthreshold signals for saccadic decisionmaking (Phongphanphanee et al., 2014), a lot more work needs to be done to conclusively show that a STOP-and-restart mechanism is unnecessary in the decision making process. An experimental study in that direction was recently published by Everling and colleagues and challenges the (prevailing) idea of a suppressive/inhibitory influence (STOP signal in the Noorani and Carpenter model) 
of prefrontal cortical areas on reflexive, erroneous prosaccade generation in this paradigm (Everling and Johnston, 2013 for a recent review).

\section{REFERENCES}

Brown, J. W., Bullock, D., and Grossberg, S. (2004). How laminar frontal cortex and basal ganglia circuits interact to control planned and reactive saccades. Neural Netw. 17, 471-510. doi: 10.1016/j.neunet.2003. 08.006

Cutsuridis, V. (2010). "Neural accumulator models of decision making," in Brain Inspired Cognitive Systems 2008, Advances in Experimental Medicine and Biology 657, eds A. Hussain, I. Aleksander, L. Smith, R. Chrisley, A. K. Barros, and V. Cutsuridis (New York, NY: Springer Science+Business Media, LLC), 61-72.

Cutsuridis, V., Kumari, V., and Ettinger, U. (2014). Antisaccade performance in schizophrenia: a neural model of decision making in the superior colliculus. Front. Neurosci. 8:13. doi: 10.3389/fnins.2014.00013

Cutsuridis, V., Smyrnis, N., Evdokimidis, I., and Perantonis, S. (2007). A neural network model of decision making in an antisaccade task by the superior colliculus. Neural Netw. 20, 690-704. doi: 10.1016/j.neunet.2007.01.004

Evdokimidis, I., Smyrnis, N., Constantinidis, T. S., Stefanis, N. C., Avramopoulos, D., Paximadis, C., et al. (2002). The antisaccade task in a sample of 2006 young men I. Normal population characteristics. Exp. Brain Res. 147, 45-52. doi: 10.1007/s00221-002-1208-4

Everling, S., and Johnston, K. (2013). Control of the superior colliculus by the lateral prefrontal cortex. Philos. Trans. R. Soc. Lond. $B$ Biol. Sci. 368:20130068. doi: 10.1098/rstb. 2013.0068

Hallett, P. E. (1978). Primary and secondary saccades to goals defined by instructions. Vis. Res. 18, 1279-1296. doi: 10.1016/0042-6989(78) 90218-3

Munoz, D. P., and Everling, S. (2004). Look away: the anti-saccade task and the voluntary control of eye movement. Nat. Rev. Neurosci. 5, 218-228. doi: 10.1038/nrn1345

Munoz, D., and Wurtz, R. (1995). Saccade related activity in monkey superior colliculus. I. Characteristics of burst and buildup cells. J. Neurophys. 73, 2313-2333.

Noorani, I., and Carpenter, R. H. S. (2013). Antisaccades as decisions: LATER model predicts latency distributions and error responses. Eur. J. Neurosci. 37, 330-338. doi: 10.1111/ejn.12025

Noorani, I., and Carpenter, R. H. S. (2014). Restarting a neural-race: anti-saccade correction. Eur. J. Neurosci. 39, 159-164. doi: 10.1111/ejn.12396

Phongphanphanee, P., Marino, R. A., Kaneda, K., Yanagawa, Y., Munoz, D. P., and Isa, T. (2014). Distinct local circuit properties of the superficial and intermediate layers of the rodent superior colliculus. Eur. J. Neurosci. 40, 2329-2343. doi: 10.1111/ejn.12579

Polsky, A., Mel, B. W., and Schiller, J. (2004). Computational subunits in thin dendrites of pyramidal cells. Nat. Neurosci. 7, 621-627. doi: 10.1038/nn 1253

Takahashi, M., Sugiuchi, Y., Izawa, Y., and Shinoda, Y. (2005). Commissural excitation and inhibition by the superior colliculus in tectoreticular neurons projecting to omnipause neuron and inhibitory burst neuron regions. J. Neurophysiol. 94, 1707-1726. doi: 10.1152/jn. 00347.2005

Conflict of Interest Statement: The author declares that the research was conducted in the absence of any commercial or financial relationships that could be construed as a potential conflict of interest.

Received: 03 October 2014; accepted: 07 January 2015; published online: 29 January 2015.

Citation: Cutsuridis V (2015) Neural competition via lateral inhibition between decision processes and not a STOP signal accounts for the antisaccade performance in healthy and schizophrenia subjects. Front. Neurosci. 9:5. doi: 10.3389/fnins.2015.00005

This article was submitted to Decision Neuroscience, a section of the journal Frontiers in Neuroscience.

Copyright (c) 2015 Cutsuridis. This is an open-access article distributed under the terms of the Creative Commons Attribution License (CC BY). The use, distribution or reproduction in other forums is permitted, provided the original author(s) or licensor are credited and that the original publication in this journal is cited, in accordance with accepted academic practice. No use, distribution or reproduction is permitted which does not comply with these terms. 